\title{
Coordination and chemical effects on the structural, electronic and magnetic properties in Mn pnictides
}

\author{
A. Continenza, S. Picozzi \\ Istituto Nazionale di Fisica della Materia (INFM) Dipartimento di Fisica, \\ Università degli Studi di L'Aquila, I-67010 Coppito (L'Aquila), Italy \\ W. T. Geng, and A. J. Freeman \\ Department of Physics and Astronomy and Materials Research Center \\ Northwestern University, Evanston, IL 60208 (U.S.A.)
}

\begin{abstract}
Simple structures of MnX binary compounds, namely hexagonal NiAs and zincblende, are studied as a function of the anion $(\mathrm{X}=\mathrm{Sb}, \mathrm{As}, \mathrm{P})$ by means of the all-electron FLAPW method within local spin density and generalized gradient approximations. An accurate analysis of the structural, electronic and magnetic properties reveals that the cubic structure greatly favours the magnetic alignment in these compounds leading to high magnetic moments and nearly half-metallic behaviour for MnSb and MnAs. The effect of the anion chemical species is related to both its size and the possible hybridization with the Mn $d$ states; both contributions are seen to hinder the magnitude of the magnetic moment for small and light anions. Our results are in very good agreement with experiment - where available - and show that the generalized gradient approximation is essential to correctly recover both the equilibrium volume and magnetic moment.
\end{abstract}

PACS numbers 71.20.-b,75.30.-m,75.50.-y 


\section{INTRODUCTION}

The renewed interest in diluted magnetic semiconductors 12 as promising materials for innovative spin-based devices, is motivating studies in many related materials. In fact, the addition of the spin degree of freedom to conventional electronics know-how is paving the way to the appealing field dubbed "spintronics". In this work, we show results obtained from first principles FLAPW calculations for Mn compounds. In particular, we look at the structurally simple binary compounds, MnSb, MnAs and MnP, and perform full optimization of the structural parameters in the hexagonal ferromagnetic NiAs phase, as well as for the more simple zincblende structure. We remark that MnP is not stable in the NiAsphase; however, its equilibrium phase (namely the MnP-type) has a crystal structure which slightly distorts from the NiAs. Moreover, while MnSb and MnAs are both ferromagnetic in their equilibrium structure, $\mathrm{MnP}$ shows a complex antiferromagnetic helical alignment at low temperatures (below 47K), is ferromagnetic between 47 and $291 \mathrm{~K}$ and paramagnetic at higher temperatures 3 .

The goal of the present work is to shed light on how $i$ ) different coordination (i.e. distorted octahedra in NiAs-type and tetrahedral in zincblende) and ii) different bonding properties (along the series MnSb, MnAs and MnP) affect the structural, electronic and magnetic properties of these Mn compounds. Moreover, we focus on how well the investigated properties are reproduced within the local spin density (LSD) and generalized gradient approximation (GGA). None of the investigated compounds is stable in the zincblende structure; however, since Mn often substitutes for the group III element in III-V compounds, it is interesting to understand and compare the electronic and magnetic properties of Mn in this coordination. In addition, it is well established that the zincblende diluted $\mathrm{Ga}_{1-x} \mathrm{Mn}_{x} \mathrm{As}_{\mathrm{s}}$ system is stable for concentrations up to $\mathrm{x}=7 \%$ and recent experiments 1 on the MBE epitaxial growth of $\mathrm{MnSb}$ (in the NiAs phase) on GaSb reported the presence of another magnetic phase showing an anomalous Hall effect - attributed to clusters of zincblende (Ga,Mn)Sb.

The paper is organized as follows: in Sect. [I] we describe few details of the calculation; 
we discuss results regarding the structural, electronic and magnetic properties on Sect. [II, IVA and $\nabla$, respectively. Finally, we draw our conclusions in Sect. V].

\section{METHOD}

All calculations were performed using the all-electron full-potential linearized augmented plane wave (FLAPW) method within the local spin density approximation to density functional theory. In order to check the accuracy, we performed structural optimizations using both LSD as parametrized by von Barth and Hedin 6 and the generalized gradient approximation (GGA) as proposed by Perdew and Wangl. We used the following muffin-tin sphere radii $R_{M n}, R_{S b}=2.40 a . u ., R_{A s}=2.0 a . u ., R_{P}=1.9$ a.u.; well converged LAPW wavefunctions were achieved using a plane-wave cutoff $k_{\max }=3.0$ a.u., resulting in about 300 basis functions (in the unit cells described below). Inside the muffin-tin spheres, we used an angular momenta expansion up to $l_{\max }=8$ for the potential and charge density representations; the same value $l_{\max }=8$ was used for the wave-function expansion. Integrations over the irreducible Brillouin zone wedge were performed using $24 k$-points according to the Monkhorst-Pack scheme目; the accuracy of this mesh has been checked by comparison with results obtained with higher numbers of inequivalent $k$-points.

\section{STRUCTURAL RESULTS}

Our results are summarized in Table 1 and Table प] for the NiAs (space group $P_{6_{3}} / m m c-$ $D_{6 h}^{4}$ - No. 194 in the International Tables for X-Ray Crystallography) and the zincblende

phase, respectively, and compared with experiments 910 and other calculations 10 . 2 . It is clear that GGA gives the best agreement with experiment (available only for $\mathrm{MnSb}$ and $\mathrm{MnAs}$ ) recovering in both cases the correct volume and shape of the unit cell. On the other hand, LSD severely underestimates the equilibrium volumes (by as much as $20 \%$ ), so that its use seems not to be very suitable when dealing with equilibrium properties of Mn pnictides. The same features have been previously observed for other compounds containing $\mathrm{Mn}^{13}$ and 
in the more studied case of $\mathrm{F}^{14}$. The equilibrium volumes for the hexagonal structures follow the trend of the anion ionic radii $\left(R_{S b}=1.40 \AA, R_{A s}=1.20 \AA, R_{P}=1.06 \AA\right)$ : we find about a $20 \%$ variation along the series which leads to about a $7 \%$ variation of the Mn-anion distance. However, due to the different $c / a$ ratios, the cell shape also changes: while the volume variaton is considerable, the value of the $c$ axis of the cell varies only by $4 \%$ from Sb to As and by $1 \%$ from As to P. In fact, the vertical size of the cell seems to be almost fixed by the Mn-Mn nearest neighbour distance $\left(d_{M n-M n}=2.89,2.84\right.$, and $2.68 \AA$ in $\mathrm{MnSb}$, MnAs and MnP, respectively). Most of the volume variation is therefore due to a shrinking of the cell basal plane which contains the anion (sitting in the middle of the hexagonal 2-dimensional cell) and determines the Mn-anion distance.

It is useful to recall that in its stable equilibrium phase, MnP shows an Mn-Mn distance $\left(d_{M n-M n}=2.69 \AA \sqrt[15]{15}\right)$ that is very close to the one we found: therefore the structure we considered is qualitatively not very far from the equilibrium one. Regarding the magnetic ordering, we note that as expected ferromagnetism is not favoured at small Mn-Mn distances: GGA -LMTO calculation $\$ 13$ on different bulk Mn phases showed that for characteristic Wigner Seitz radii $\left(r_{W S} \simeq 1.4 \AA\right.$, corresponding to $\left.d_{M n-M n} \simeq 2.8 \AA\right)$ the most stable alignment is antiferromagnetic; this might confirm the reason why the ferromagnetic NiAs phase is not stable for $\mathrm{MnP}$.

Let us now consider the same compounds in the zincblende structure. We think it is worthwhile stressing the relevance of this point, since many of the novel magnetic semiconductors proposed so far are compounds derived by their parent non-magnetic semiconductor in this latter phase, in which even large concentrations (up to 50\%16) of cations have been substituted by Mn. As a result, the final coordination of the Mn atoms is strictly tetrahedral.

Our results obtained using the GGA approximation are reported on Table III. Due to difficulties in stabilizing the cubic phase, experimental data for zincblende MnX compounds are not available; however, an experimental study $\left[7\right.$ focused on a $\mathrm{Ga}_{1-x} \mathrm{Mn}_{x} \mathrm{As}$ system with Mn concentrations up to $x=0.07$, showed a linear extrapolation at $x=1$ of the alloy lattice constant (assuming Vegard's law validity) leading to $a=5.87 \AA$. This differs by $3.7 \%$ from 
our result for MnAs; however, we must remark that the linear extrapolation may indicate an incorrect value, since the actual experimental lattice constant data are reported only for very small Mn concentrations. Moreover, recent calculations 18 pointed out significant deviations from Vegard's rule in $\mathrm{Ga}_{x} \mathrm{Mn}_{1-x}$ As systems. Other first principles calculations of the MnAs lattice constant performed without GGA give $a=5.66$ 国 and $a=5.87$ A 9 within a pseudopotential and FLAPW formalism, respectively. The differences with our LDA value (not shown in the tables, $a=5.34 \AA$ ) are probably to be ascribed to the use of pseudopotential approach in the first case and to a different minimization approach or FLAPW implementation in the second case. It needs to be pointed out, however, that a reliable determination of the equilibrium lattice constant is particularly important for the corresponding magnetic properties. In fact, as explained in detail below, the magnetic moment shows a strong dependence on the volume: an overestimate of the equilibrium zincblende lattice constant may even lead to an incorrect stable half-metallic behaviour. Furthermore, from Table $\mathbb{\square}$ we note that the equilibrium volume per formula unit (i.e. per $\mathrm{MnX}$ pair) scales with the anion ionic radii in both phases, and it is always larger in the zincblende phase (see also Fig. [1). In particular, we remark that while the Mn-anion bond length is very similar in the NiAs and zincblende phase (only $4 \%$ larger in the cubic phase), the Mn-Mn nearest neighbour distances are very much larger in the zincblende phase $\left(d_{M n-M n}=4.36,3.99\right.$, and $3.75 \AA$ for $\mathrm{MnSb}, \mathrm{MnAs}$ and $\mathrm{MnP}$, respectively). These large distances favour ferromagnetic alignment and the direct exchange interaction, leading to an enhanced magnetic moment. It is very interesting to note in Table [1 the very good matching conditions between the $\mathrm{MnX}$ zincblende and its Ga-based analogue: this suggests that under non-equilibrium conditions Mn may easily occupy the cation sites, if the growth is achieved on suitable substrates and if solubility problems are overcome.

In order to show the stability of the different phases, the total energy behaviour versus volume/formula-unit is plotted in Fig. 1. On the same plots we also report the total magnetic moment as a function of the volume. In all the compounds analyzed, the NiAs-phase is more stable than the zincblende phase by more than $0.7 \mathrm{eV}$ per formula unit. In addition, the 
zincblende structure shows a larger equilibrium volume with respect to the NiAs phase (30\% in $\mathrm{MnSb}$ and $25 \%$ in $\mathrm{MnP}$ ) joined by a parallel increase in the magnetic moment per cell. However, the NiAs phase remains the most stable over a large range of pressures showing that even at the most favorable growing conditions for the zincblende phase, the NiAs phase may segregate forming clusters, as found, for example, in the growth of (Ga,Mn)As at $\mathrm{Mn}$ concentrations higher than $7 \%$ 2 2 .

\section{ELECTRONIC PROPERTIES}

\section{A. NiAs phase}

We now discuss the electronic properties of the MnX compounds in both phases. In Fig.2, we plot the band dispersion for the NiAs phase along the $\Gamma-\mathrm{A}$ direction. The band dispersion along this line is very similar in all three compounds considered. At high binding energies, we find the anion- $s$ derived bands: these states are very little affected by the Mn exchange energy and follow the expected trend in energy related to the atomic s-levels $(5 \mathrm{~s}$, 4s, and 3s for $\mathrm{Sb}, \mathrm{As}$ and $\mathrm{P}$, respectively). At energies closer to the Fermi level $\left(E_{F}\right)$, we find the Mn $d$ bands hybridized with the anion $-p$ states. Actually, the lower states $(\sim-5.0 \mathrm{eV})$ in this group are those with higher $p$ hybridization (still less than 7-8 \%), while the others have Mn $d$ character exceeding $48 \%$ at $\Gamma$. A somewhat larger hybridization is allowed by symmetry at the A point, reaching about $12 \%$ for states well below the Fermi level $\left(E_{F}\right)$ for the majority spin component (i.e. states with $A_{3}$ symmetry bonding and non-bonding as indicated in Fig. 2 by the solid and dashed bold lines, respectively).

The states crossing $E_{F}$ show high anion $p$ character at $\Gamma$ (up to $20 \%$ ), but become entirely Mn $d$ states at A. For the minority component we can easily trace the same features, shifted at lower binding energies due to the exchange interaction. This latter determines only a partial and small occupation of the lower non-bonding $A_{3}$ split band for $k$-vectors very close to the zone center for MnSb and MnAs, while in MnP we find a complete half-filling of the 
4-fold degenerate $A_{3}$ state. Looking at the series $\mathrm{Sb}, \mathrm{As}$, $\mathrm{P}$, we can see that the states at $A$ denoted by the bold solid lines in Fig. 2 are very close in the Sb compound while their separation increases as we lower the anion atomic number; at the same time we find that the exchange splitting (i.e. the energy separation between corresponding states for the majority and minority component) decreases leading to the partial filling of the higher $A_{3}$ state in MnP. Moreover, at $\Gamma$ the symmetry allowed $p-d$ hybridization is seen to increase the band width of the states originating from the lower $A_{3}$ state (bold solid line in Fig. 2) on going from $\mathrm{MnSb}$ to $\mathrm{MnP}$. On the other hand, at the $A$ point we find a slightly lower anion $p$ component for $\mathrm{MnP}(7 \%)$ compared to $\mathrm{MnSb}(12 \%)$, probably related to the smaller sphere used for P.

The picture that emerges from the band structure is the following: while MnSb and MnAs show a very similar $p-d$ hybridization, the compound with $\mathrm{P}$ seems to have a higher hybridization which affects the exchange splitting of the Mn $d$ states. This is more clearly seen by inspection of the density of states projected (PDOS) on each atomic site. In Fig. 因, we show the PDOS for MnSb and MnP (in particular Mn $d$ states and anion $s$ and $p$ states: analogous data for the MnAs compound are not reported since they are half-way between the two limiting $\mathrm{MnSb}$ and $\mathrm{MnP}$ cases). In $\mathrm{MnP}$, we found a broader valence band: the $\mathrm{P}$ $p$ states start at around $-7.5 \mathrm{eV}$ and give rise to two well separated peaks; the feature at lower binding energies $(\sim-3.0 \mathrm{eV})$ is due to strong hybridization with the $\mathrm{Mn} d$ states that causes a splitting of the $d$ band. These two different features can be traced back to the $A_{3}$ states (see Fig.2) discussed previously (in MnSb these states are very close in energy in the majority component while in $\mathrm{MnP}$ they are split by about $1.5 \mathrm{eV}$ ). The higher hybridization in $\mathrm{MnP}$ leads to a broadening of the $\mathrm{Mn} 4 d$ band joined by a smaller effective exchange energy among $d$ states and a consequent higher filling of the minority component.

In order to complete the discussion of the electronic properties, we compare our calculated GGA results with available photoemission data and other calculations in Tables $\mathbb{1 1 T}$ and $\mathbb{I V}$. The agreement between experimenta 20 and theoretical data is noticeably improved by the present GGA calculations in MnSb, for which there are detailed ARUPS data available; 
we expect that the same agreement might hold for the other compounds. For MnAs, we compare with UPS spectra2n which, however, are not very detailed; experiments find a broader Mn $d$ band with respect to $\mathrm{MnSb}$ centered around $2 \mathrm{eV}$, and this is consistent with our calculation. Also, the tail structure observed in the MnAs case at 4-6 eV below $E_{F}$ is consistent with our PDOS (not shown) which shows a broadening due to $p-d$ hybridization in-between MnSb and MnP case.

For $\mathrm{MnP}$, we can compare with recent UPS and IPES data $3^{3}$ which find (for the real MnP-type structure) a broader Mn $d$ band compared to Sb, a small peak at $-0.4 \mathrm{eV}$ and broad structures at $-2.6,-6.4$ and $-10 \mathrm{eV}$. These data match very well the features found in our calculations and shown in Fig.4: very close to $E_{F}$ we find a small feature related to Mn $d$ states hybridized with $\mathrm{P} p$ states corresponding to the band crossing $E_{F}$ and which is very flat at the $A$ point (compare with Fig.尹). At higher binding energies (from $E_{F}$ down to $3 \mathrm{eV}$ ) the $\mathrm{Mn} d$ contribution is predominant while the feature at $6 \mathrm{eV}$, according to our results, has to be ascribed to $\mathrm{P} p$ states. In agreement with our calculations, photoemission experiments find a much smaller $d$ exchange split energy (i.e. the energy distance between occupied and unoccupied $d$ states) in $\mathrm{MnP}$ compared to $\mathrm{MnSb}$ (about $2 \mathrm{eV}$ and more than $3 \mathrm{eV}$ in $\mathrm{MnP}$ and $\mathrm{MnSb}$, respectively).

We point out that after including relativistic corrections, namely spin-orbit coupling, on $\mathrm{MnSb}$ (where it is expected to play the most important role), we found that the energy splitting for the bands with higher Mn $p$ content was of the order of $0.5 \mathrm{eV}$. However, the energy levels involved (mainly the Mn $d$ states hybridized with Sb $p$ states) are at energies far from $E_{F}$ and therefore do not affect effectively the magnetic moment or the exchange interaction among Mn $d$ states, as we will see below. Therefore, we did not explicitly consider the spin-orbit coupling on the bands shown, since this would greatly complicate the plots and the discussion of the band structure without really changing the overall physics. 


\section{B. Zincblende phase}

We now discuss the electronic properties of these same compounds in the zincblende phase. The band structures are reported in Fig. 3 and the main features can once again be related to the different degree of $p-d$ hybridization. As a general characteristic, in agreement with that already found in similar calculations 11 , we find that at $\Gamma$ the lower state is a 3 - fold degenerate $\Gamma_{15}$ state $\left(t_{2 g}\right)$ which allows hybridization with $p$ states, while the doubly degenerate $\Gamma_{12}$ state $\left(e_{g}\right)$ has entirely $\mathrm{Mn} d$ character. The states with mainly anion $p$ character are above $E_{F}$ and show a very wide dispersion so that in all three compounds they give rise to electron pockets at the zone boundaries (namely at X and L). As expected, the exchange interaction is larger for the non-hybridized $\Gamma_{12}$ states and follows the same trend with hybridization as previously discussed for the NiAs phase: lighter anions have deeper lying $p$ levels (and are therefore closer in energy to the Mn $3 \mathrm{~d}$ states) so that they allow for greater hybridization. In fact, we find that while the $p / d$ occupation ratio is about 0.06 for MnSb, it becomes 0.13 in MnP. This leads to a broader $\mathrm{Mn} d$ band and significantly lowers the exchange interaction along the series, as shown in Table $\square$.

In all cases, however, the exchange splitting is higher than in the corresponding NiAs phase showing that the lower coordination greatly favors the $d$ - $d$ interaction leading to a higher magnetic moment, as will be discussed in greater detail below. The band structures for the minority components show that MnSb and MnAs are nearly half-metallic (the occupied portion of the $\Gamma_{12}$ state is very close to zero) while MnP shows a much larger occupation factor. This is of course of great interest in the design of new semiconducting magnetic materials since it shows that properly grown materials might present very appealing properties, as hinted by the study of Abe et al. on (Ga,Mn)Sb.

We can compare our results for zincblende MnAs with a photoemission study 22 performed on cubic $G a_{1-x} M n_{x} A s$. We find that the main features are nicely reproduced in our calculation for $x=1$; in particular, we find a quite broad Mn $d$ band centered at around $2.5 \mathrm{eV}$ and features around $5 \mathrm{eV}$ below $E_{F}$ that are related to As $p-\mathrm{Mn} d$ hybridization. 
Our calculations, however, do not reproduce the satellite state at $6 \mathrm{eV}$ below $E_{F}$ that seems to be related to many-body correlation effects. However, if we look at the exchange splitting for the $\Gamma_{12}$ state $(3.4 \mathrm{eV})$ we find that it is remarkably close to the Coulomb repulsion value $(\mathrm{U}=3.5 \pm 1.0 \mathrm{eV})$ obtained 22 by fitting the experimental data with a configuration interaction (CI) MnAs cluster model. Moreover, an estimate of the $p-d$ hybridization (the $p d \sigma=1.0 \pm 0.1 \mathrm{eV}$ parameter fitted in the CI approximation) can be obtained from our calculation by looking at the width of the $\Gamma_{15}$ state at the zone boundary $(1.16 \mathrm{eV})$ where the lower split band gains more $p$ weight. Although our values are calculated in the very high concentration limit, the agreement with experiment should not be very surprising since the quantities we have been looking at are really characteristic of the Mn-As bond and are therefore expected not to be affected by the presence of Ga.

\section{MAGNETIC PROPERTIES}

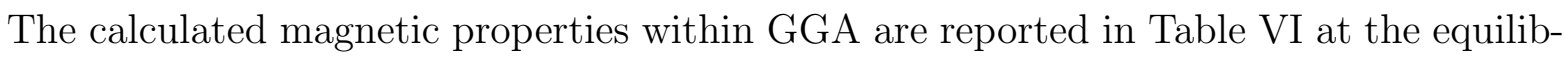
rium volume for both phases considered. The corresponding values calculated within LSD are not reported, since the equilibrium volumes are not well reproduced; the LSD calculated magnetic moments at fixed volume were always smaller (about 5\%) than the corresponding GGA values. We should remark that the values in Table V1 take into account the spincontribution only; as already mentioned, in fact, spin-orbit inclusion did not significantly affect the total spin magnetic moment (by less than $0.1 \mu_{B}$ in the Sb case). We also did not

consider any orbital contribution to the total magnetic moment 23 or the effect of magnetic anisotropy related to different quantization axes: the effects have been shown23 to be of the order of 0.04 and $0.02 \mu_{B}$, for MnSb and MnAs, respectively. We are interested in trends and in understanding how the different coordination may affect the magnetic properties more than in reproducing the exact magnetic behaviour. Moreover, in cubic compounds we expect these effects to play an even smaller role due to symmetry.

As already pointed out, in all structures an opposite magnetic alignment with respect to 
$\mathrm{Mn}$ is present on the anion sites, whose contribution decreases in going from the hexagonal to the cubic structure. This characteristic is confirmed by experimental observations ${ }^{24}$ in both $\mathrm{MnSb}$ and MnAs and can be traced back to the $p$ - $d$ hybridization. In particular, in $\mathrm{MnSb}$ the total magnetic moment of $3.5 \mu_{B}$ with a spherically symmetric magnetic moment of -0.2 $\mu_{B}$ at the $\mathrm{Sb}$ site is reporteden, while in MnAs a magnetic moment of $3.4 \mu_{B}$ per formula unit with $-0.23 \mu_{B}$ on the As sites has been measured by neutron scattering experiments. The situation in $\mathrm{MnP}$ is by far more complex due to the particular structure and the noncollinear magnetism found in this compound. Looking at the trends along the series $\mathrm{Sb} \rightarrow$ As $\rightarrow \mathrm{P}$, Mn shows a higher magnetic moment in the zincblende structure due to the smaller hybridization with the anion $p$ states in this symmetry; as we mentioned earlier, only the $\Gamma_{15}$ state symmetry allows for $p$ hybridization, while the $\Gamma_{12}$ symmetry is not compatible with any $p$ character. This enhances the exchange interaction for these states, whose minority counterpart is found above $E_{F}$ and is therefore almost entirely unoccupied (e.g. MnSb and MnAs) leading to a nearly half-metallic behaviour.

As a result, we find that there are two different mechanisms that have to be considered when analyzing the trends along the series $\mathrm{Sb}$, As and $\mathrm{P}: i$ ) a structural effect related to the decreasing anion size and ii) a chemical effect which changes the state hybridization. Both contributions result in a reduction of the magnetic properties as the anion becomes smaller; in fact, the smaller size leads to a volume reduction and to a consequent smaller Mn-Mn bond length which increases the $d-d$ overlap and reduces the exchange interaction. At the same time, the $p$ - $d$ hybridization, which is favoured for lighter anions, also lowers the $d$ - $d$ exchange interaction, therefore reducing the resulting magnetic moment. These same contributions are found in both phases and play the same role in both coordinations; however, the zincblende phase is seen to be the most favorable for enhanced magnetic properties since, due to symmetry, it matches both requirements: larger volume and reduced hybridization. 


\section{CONCLUSIONS}

We presented results on the structural and electronic properties of MnSb, MnAs and $\mathrm{MnP}$ in two different coordinations: hexagonal NiAs and zincblende. Our results show that LSD is not well suited to correctly describe the structural properties of these compounds since it severely underestimates the equilibrium volumes. Moreover, at a fixed volume, the calculated spin contribution to the magnetic moment within LSD is about $5 \%$ smaller than the one calculated within GGA.

Comparing our calculated results with available experiments, we found very good agreement for the structural properties as well as for the electronic properties: our findings match very closely photoemission data and ARUPS results, where available. Moreover, from a detailed analysis of these compounds in different phases, we were able to establish that the cubic coordination greatly favours the magnetic alignment leading to quite high magnetic moments due to $i$ ) lower $p$ - $d$ hybridization, and $i i$ ) larger equilibrium volumes.

On the same footing, we found that in the search for suitable compounds with high magnetic alignment, the heavier anions should be preferred since they form compounds with larger volume and lower $p-d$ hybridization.

\section{ACKNOWLEDGEMENTS}

Work at Northwestern University supported by the NSF (through the Materials Research Center). 


\section{TABLES}

TABLE I. Calculated structural parameters for $\mathrm{MnSb}$, MnAs and $\mathrm{MnP}$ in the NiAs phase compared with available experiments. The magnetic moment, $\mu$, is calculated within the muffin-tin sphere.

\begin{tabular}{|c|ccc|ccc|ccc|}
\hline \hline & \multicolumn{5}{|c|}{ MnSb } & \multicolumn{3}{c|}{ MnAs } & \multicolumn{3}{c|}{ MnP } \\
\hline & $a$ & $c / a$ & $\mu$ & $a$ & $c / a$ & $\mu$ & $a$ & $c / a$ & $\mu$ \\
& $(\AA)$ & & $\left(\mu_{B}\right)$ & $(\AA)$ & & $\left(\mu_{B}\right)$ & $(\AA)$ & & $\left(\mu_{B}\right)$ \\
\hline \hline \multirow{2}{*}{$\begin{array}{c}\text { this work LSD } \\
\text { this work GGA }\end{array}$} & 3.863 & 1.37 & 2.46 & 3.487 & 1.49 & 1.91 & - & - & - \\
Expt. & 4.128 & 1.35 & 3.12 & 3.704 & 1.49 & 3.15 & 3.386 & 1.62 & 2.04 \\
\hline \hline
\end{tabular}

${ }^{a}$ Ref. 9

${ }^{b}$ Ref. 10

${ }^{c}$ Ref. 12

TABLE II. Calculated equilibrium lattice constants and magnetic moments for MnSb, MnAs and $\mathrm{MnP}$ within GGA for the zincblende phase compared with the experimental lattice constants of the related zincblende Ga compounds.

\begin{tabular}{|c|c|c|c|}
\hline \hline $\mathrm{X}$ & $\mathrm{a}_{M n X}(\AA)$ & $\mathrm{a}_{G a X}(\AA)$ & $\mu_{M n X}\left(\mu_{B}\right)$ \\
\hline $\mathrm{Sb}$ & 6.166 & 6.096 & 3.77 \\
$\mathrm{As}$ & 5.643 & 5.657 & 3.75 \\
$\mathrm{P}$ & 5.308 & 5.451 & 2.73 \\
\hline \hline
\end{tabular}


TABLE III. Calculated exchange splittings (in eV) and magnetic moment for MnSb compared with other calculations and experiments.

\begin{tabular}{|c|c|c|c|c|}
\hline & expt. $^{a}$ & $\mathrm{ASW}^{b}$ & FLAPW $^{c}$ & present work GGA \\
\hline$\Delta_{e x}(\Gamma$, bond. $)$ & $1.4 \pm 0.3$ & 1.51 & 1.36 & 1.43 \\
\hline$\Delta_{e x}(\mathrm{~A}$, bond. $)$ & $1.7 \pm 0.3$ & 1.72 & 1.56 & 1.67 \\
\hline$\Delta_{e x}(\Gamma$, non-bond $)$ & $\sim 3.0$ & 2.51 & 2.48 & 2.88 \\
\hline$\Delta_{e x}(\mathrm{~A}$, non-bond. $)$ & $>2.8$ & 2.66 & 2.62 & 3.01 \\
\hline$\mu$ & 3.5 & 3.24 & 3.21 & 3.36 \\
\hline
\end{tabular}

${ }^{a}$ Ref. 20 .

${ }^{b}$ Ref. 11.

${ }^{c}$ Ref. 21 .

TABLE IV. Calculated exchange splittings (in eV) and magnetic moment for MnSb, MnAs and $\mathrm{MnP}$ in the NiAs phase.

\begin{tabular}{|c|c|c|c|c|}
\hline \hline & $\Delta_{e x}(\Gamma$, bond. $)$ & $\Delta_{e x}(\Gamma$, non - bond $)$ & $\Delta_{e x}(\mathrm{~A}$, bond. $)$ & $\Delta_{e x}$ (A, non-bond.) \\
\hline \hline $\mathrm{MnSb}$ & 1.43 & 2.88 & 1.67 & 3.01 \\
$\mathrm{MnAs}$ & 1.397 & 2.87 & 1.60 & 3.02 \\
$\mathrm{MnP}$ & 0.775 & 1.757 & 0.905 & 1.87 \\
\hline \hline
\end{tabular}

TABLE V. Calculated exchange splittings (in eV) and magnetic moment for MnSb, MnAs and $\mathrm{MnP}$ in the zincblende phase.

\begin{tabular}{|c|r|r|}
\hline \hline & $\Delta_{e x}\left(\Gamma_{15}\right)$ & $\Delta_{e x}\left(\Gamma_{12}\right)$ \\
\hline \hline $\mathrm{MnSb}$ & 2.01 & 3.61 \\
$\mathrm{MnAs}$ & 1.60 & 3.40 \\
$\mathrm{MnP}$ & 1.16 & 2.83 \\
\hline \hline
\end{tabular}


TABLE VI. Calculated spin magnetic moments (in $\mu_{B}$ ) for MnSb, MnAs and MnP in the NiAs and zincblende phase

\begin{tabular}{|c|ccc|ccc|}
\hline \hline & & NiAs & & & zincbl. \\
& $\mu_{M n}$ & $\mu_{X}$ & $\mu_{T}$ & $\mu_{M n}$ & $\mu_{X}$ & $\mu_{T}$ \\
\hline \hline $\mathrm{MnSb}$ & 3.41 & -0.14 & 3.26 & 3.86 & -0.09 & 3.77 \\
$\mathrm{MnAs}$ & 3.18 & -0.10 & 3.08 & 3.83 & -0.08 & 3.75 \\
$\mathrm{MnP}$ & 2.16 & -0.08 & 2.08 & 2.79 & -0.06 & 2.73 \\
\hline \hline \hline
\end{tabular}




\section{FIGURES}

FIG. 1. Energy versus volume (solid lines) and Mn magnetic moment (dashed lines) versus volume for the $\mathrm{MnX}$ compounds ( $\mathrm{X}=\mathrm{Sb}, \mathrm{As}, \mathrm{P})$ in the NiAs (circles) and zincblende (squares) phases.

FIG. 2. Energy bands for the MnX compounds in the NiAs phase along the $\Gamma-\mathrm{A}$ symmetry line for the majority (upper panels) and minority (lower panels) spin components. The energy zero is set to the Fermi level in each compound. The thick solid (dashed) lines indicate the bonding (non-bonding) Mn-d bands.

FIG. 3. Energy bands for the MnX compounds in the zincblende phase along the $\mathrm{X}-\mathrm{\Gamma}-\mathrm{L}$ sym-

metry lines for the majority (upper panels) and minority (lower panels) spin components. The energy zero is set to the Fermi level in each compound.

FIG. 4. Density of states projected on the atomic site for $\mathrm{MnSb}$ and $\mathrm{MnP}$ in the NiAs phase. The energy zero is set to the Fermi level. 


\section{REFERENCES}

${ }^{1}$ G. Prinz, Science 282, 1660 (1998); Y. Ohno, D. K. Young, B. Beschoten, F. Matsukura, H. Ohno and D. D. Awschalom, Nature 402, 790 (1999); T. Dietl, H. Ohno, F. Matsukura, J. Cibert and D. Ferrand, Science 287, 1019 (2000).

${ }^{2}$ H. Ohno, Science, 281, 951 (1998).

${ }^{3}$ H. Okuda, S. Senba, H. Sato, K. Shimada, H. Namatame, and M. Taniguchi, Journ. Electr. Spectr. and Rel. Phenom. 101-103, 657 (1999).

${ }^{4}$ E. Abe, F. Matsukura, H. Yasuda, Y. Ohno, H. Ohno, Physica E 7, 981 (2000).

${ }^{5}$ H.J.F.Jansen, and A.J.Freeman, Phys. Rev. B 30, 561 (1984); E. Wimmer, H. Krakauer, M. Weinert, and A.J.Freeman, Phys. Rev. B 24, 864 (1981) and references therein.

${ }^{6}$ U. von Barth and L. Hedin, J. Phys. C 5, 1629 (1972).

${ }^{7}$ J.P Perdew and Y. Wang, Phys. Rev. B 45, 13244 (1992).

${ }^{8}$ H.J.Monkhorst and J.D.Pack, Phys. Rev. B 13, 5188 (1976).

${ }^{9}$ R.W.G. Wyckoff, Crystal Structures, 2nd ed. (Interscience, New York, 1960), Vol.1.

${ }^{10}$ J. Bouwma, Ph.D. dissertation, University of Groningen (1972)

${ }^{11}$ R. Coehoorn, C. Haas, and R.A. de Groot, Phys. Rev. B 31, 1980 (1985).

12 S. Sanvito and N.A. Hill, Phys. Rev B 62, 15553 (2000).

13 T. Asada, and K. Terakura, Phys. Rev. 23, 15992 (1993).

${ }^{14}$ E.G. Moroni, G. Kresse, J. Hafner, and J. Furthmuller, Phys. Rev. B 56, 15629 (1997)

${ }^{15}$ A. Yanase, and A. Hasegawa, J. Physc. C: Solid State Phys. 13, 1989 (1980).

${ }^{16}$ G.A. Medvedkin, T. Ishibashi, T.N. Hayata, Y. Hasegawa and K. Sato, Jap. Journ. of Appl. Phys. 39, 1949 (2000). 
${ }^{17}$ H. Ohno, J. Magn. Magn. Mater. 200, 110 (1999).

${ }^{18}$ Y. J. Zhao, W. T. Geng and A. J. Freeman, to be published.

${ }^{19}$ M.Shirai, T. Ogawa, I. Kitagawa, N. Suzuki, J. Magn. Magn. Mater. 177-181, 1383 (1998).

${ }^{20}$ O. Rader, A. Kimura, N. Kamakura, K. An, A. Kakizaki, S. Miyanishi, H. Akinaga, M. Shirai, K. Shimada, and A. Fujimori, Phys. Rev. B 57, R689 (1998)

${ }^{21}$ M. Shirai, Y.Tokioka, Journ. of Electr. Spectr. and Rel. Phen.88-91, 357 (1998)

${ }^{22}$ J. Okabayashi, A. Kimura, T. Mizokawa, A. Fujimori, T. Hayashi and M. Tanaka, Phys. Rev. B 59, R2486 (1999).

${ }^{23}$ P. Ravindran, A. Delin, P.James, B. Johansson, J.M. Wills, R. Ahuja, and O. Eriksson, Phys. Rev. B 59, 15680 (1999).

${ }^{24}$ Y. Yamaguchi, H. Watanabe, and T. Suzuki, J. Phys. Soc. Jpn. 45, 846 (1978); Y. Yamaguchi and H. Watanabe, J. Magn. Magn. Mater. 31-34, 619 (1983).

${ }^{25}$ K. Shimada, O. Rader, A. Fujimori, A. Kimura, K. Ono, N. Kamaakura, A. Kakizaki, M. Tanaka, and M. Shirai, J. Magn. Magn. Mater. 101-103, 383 (1999) 
Fig. 1 A. Continenza et al.

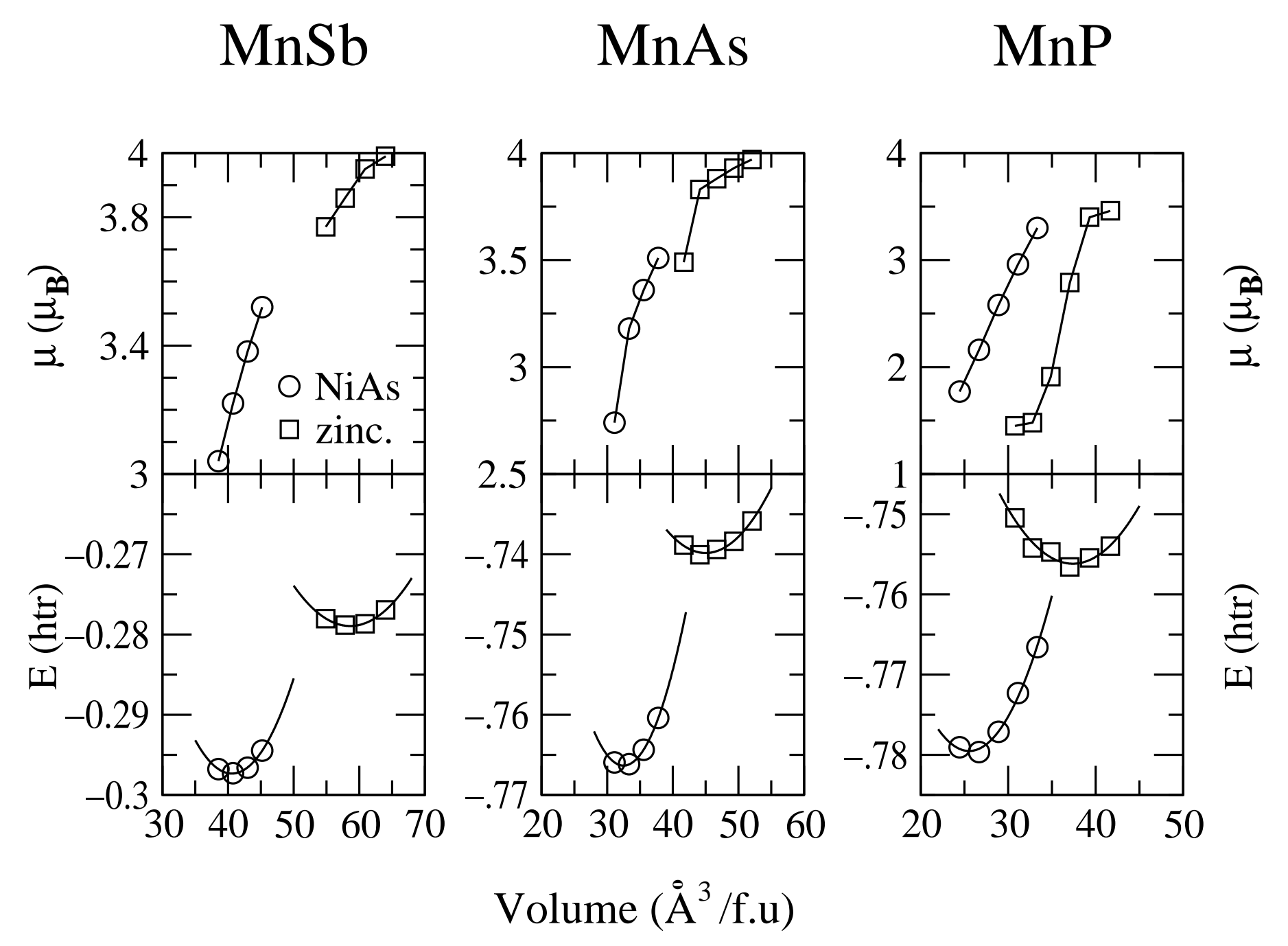


Fig.2 A. Continenza et al.

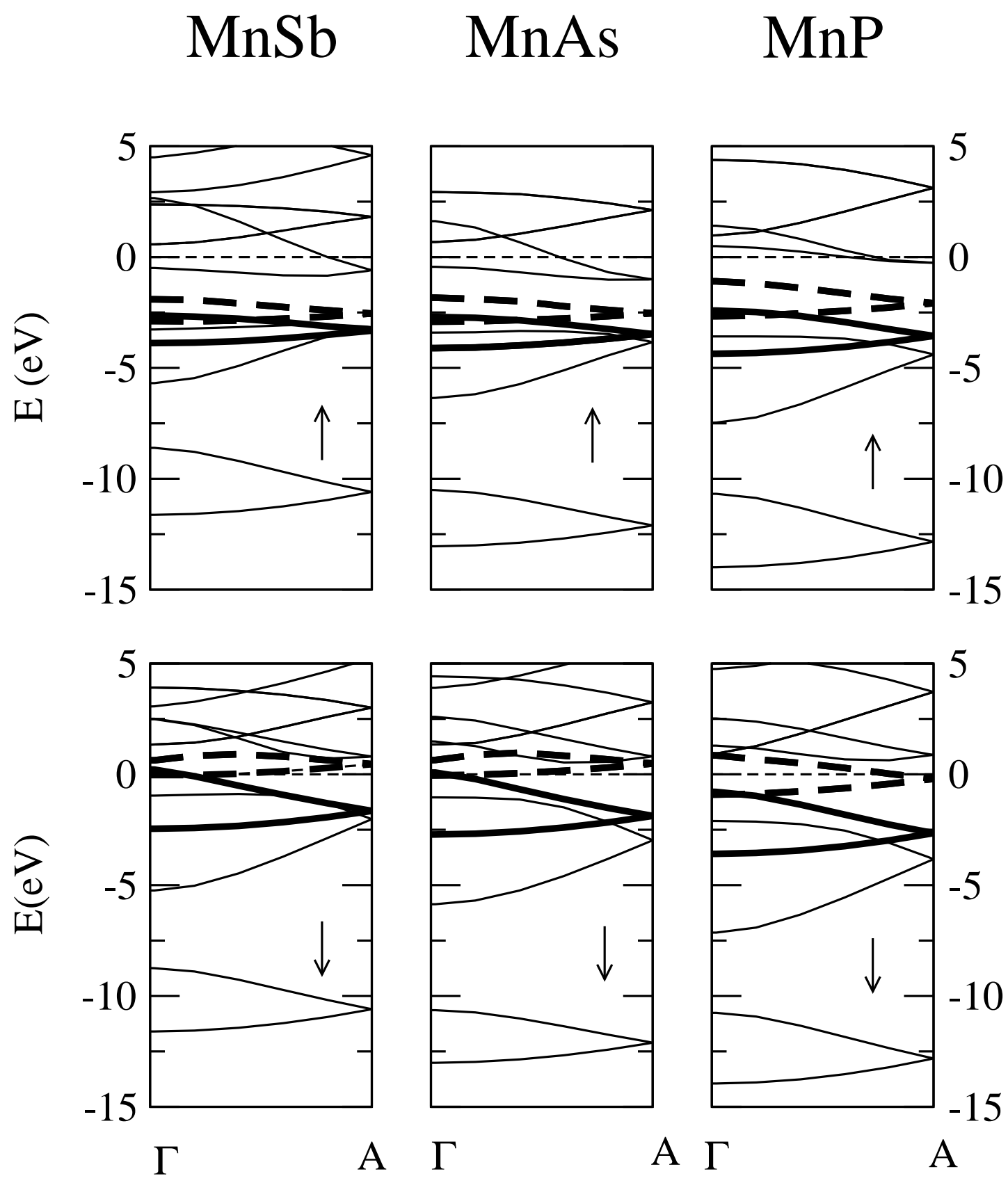


Fig.3 A. Continenza et al.

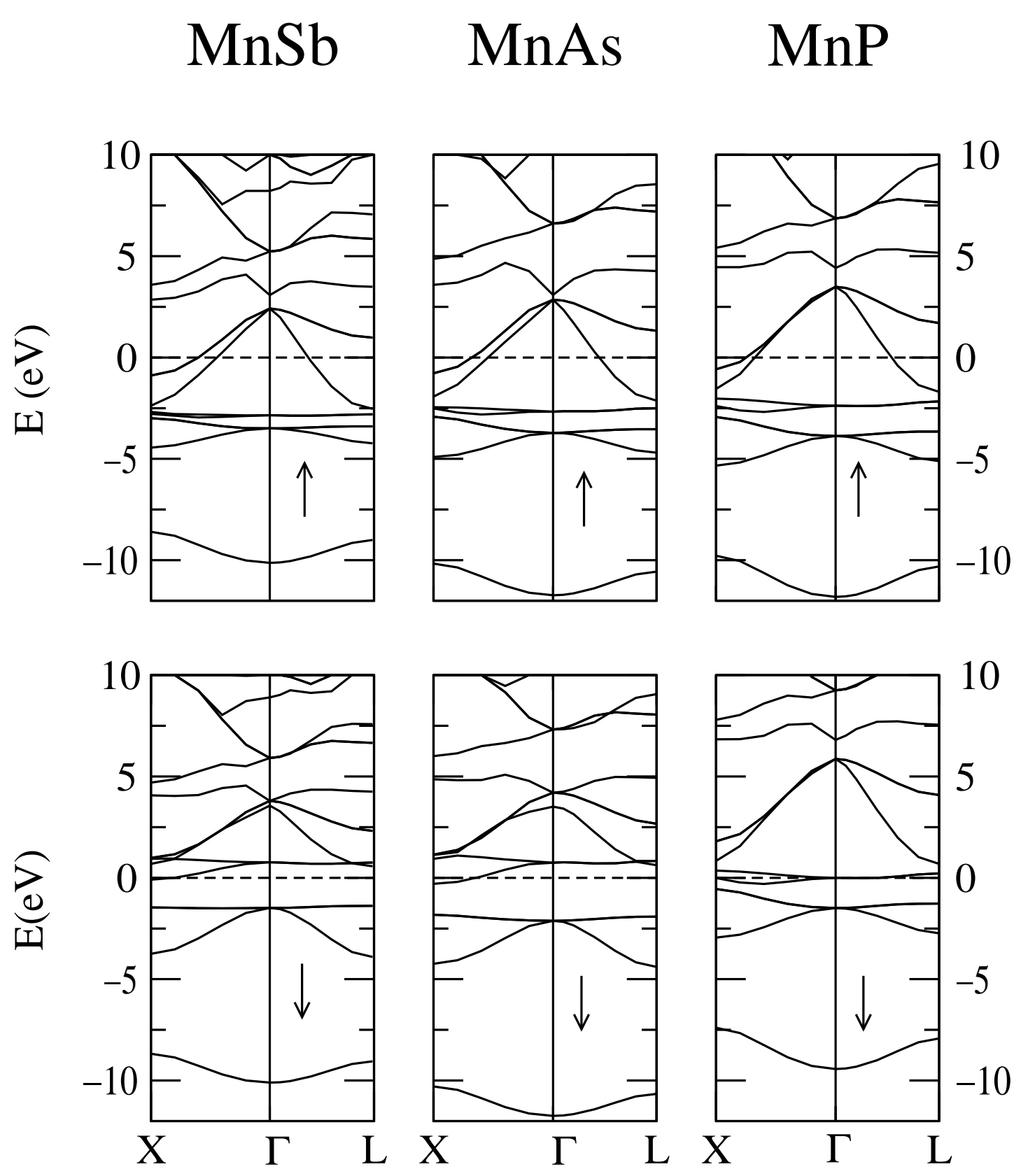


Fig. 4 A. Continenza et al.

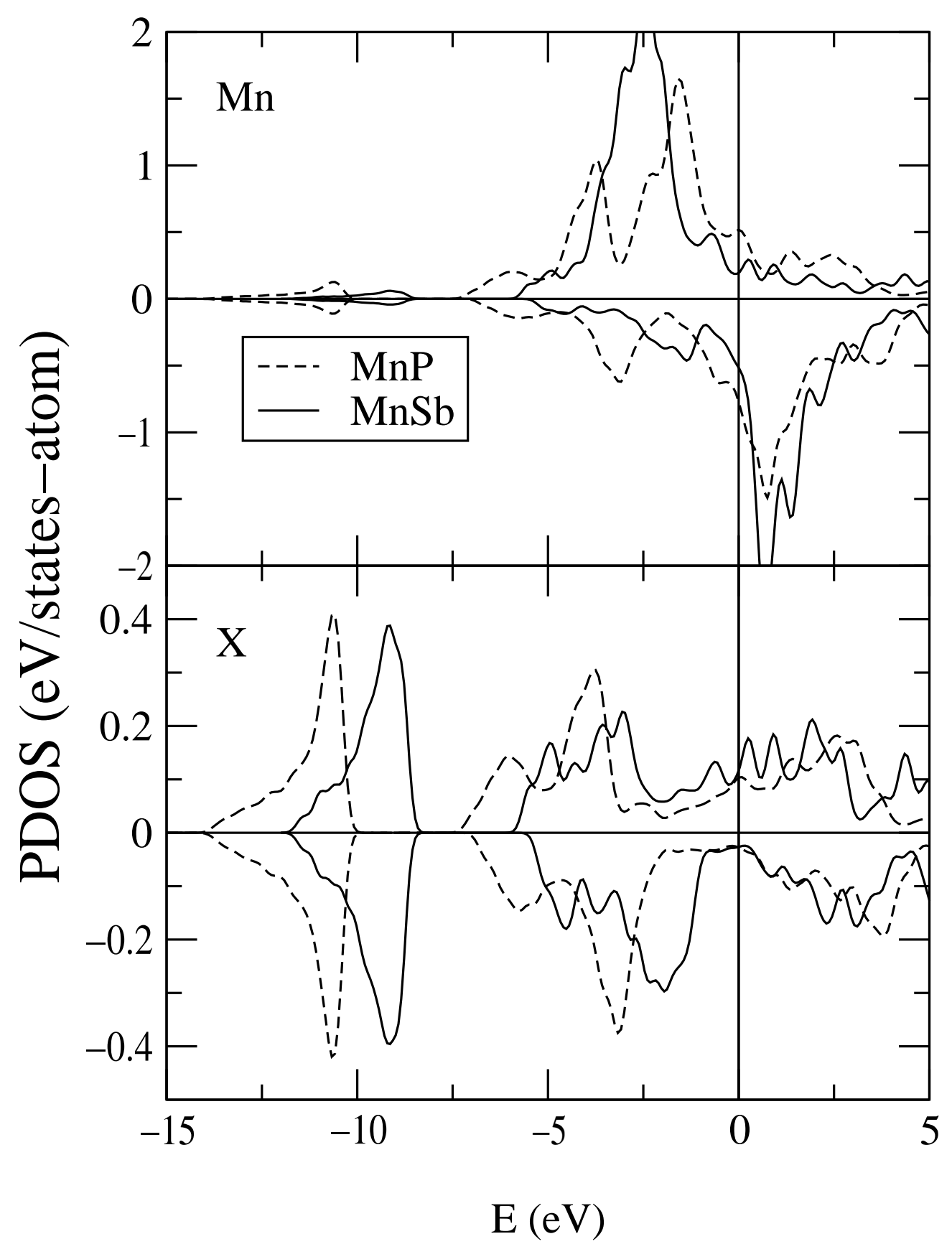

